\title{
Joule-Thomson expansion of the Bardeen-AdS black holes
}

\author{
Cong Li, Pengzhang He, Ping Li, and Jian-Bo Deng* \\ Institute of Theoretical Physics, LanZhou University, Lanzhou 730000, P. R. China
}

(Dated: June 17, 2019)

\begin{abstract}
The Joule-Thomson expansion process is studied for Bardeen-AdS black holes in the extended phase space. Firstly, we get Joule-Thomson coefficient and find that the divergent point of Joule-Thomson coefficient coincides with the zero point of temperature. The inversion curves are also obtained from the zero point of Joule-Thomson coefficient. Then the minimum inversion temperature and the corresponding mass are obtained. In addition, the ratio between minimum inversion and critical temperature for Bardeen-AdS black holes is also calculated. We obtain the isenthalpic curve in $T-P$ graph and demonstrate the cooling-heating region by the inversion curve. An interesting phenomenon we get is that black hole whose mass to charge ratio is below the critical value is always in heating process. The same phenomenon can be also obtained from the charged AdS black holes.

PACS numbers: 04.20.-q, 04.70.Dy
\end{abstract}

* Jian-Bo Deng: dengjb@lzu.edu.cn 


\section{INTRODUCTION}

Since Bekenstein and Hawking firstly studied the properties of black hole thermodynamics [1 3], thermodynamics of black holes has attracted lots of attention. Anti-de-Sitter (AdS) black hole thermodynamics has been first studied by Hawking and Page and Hawking-Page phase transition was found [4]. What's more, properties of the charged AdS black hole thermodynamics were studied in [5, 6] where a van der Waals like phase transition was found in the charged AdS black holes.

In the extended phase space, where the cosmological constant $\Lambda$ is regarded as a thermodynamic variable related to the positive pressure $P$ in AdS space, the analogy between black holes and van der Waals liquid-gas system[7] was studied and the critical exponents [8] have also been found. This analogy has been generalized to different AdS black holes, such as Kerr-black holes, Gauss-Bonnet black holes, the higher dimensional black holes and the Lovelock gravity, etc. [9-15]. It was shown in [7] that considering the cosmological constant $\Lambda$ as pressure $P$, the black hole mass $M$ was the enthalpy $H$ rather than the internal energy $U$. Furthermore, the reentrant phase transition [9, 16] and the triple point[11, 17] for AdS black holes have also been studied in the extended phase space, proposing that AdS black holes behaved similarly to the ordinary thermodynamic systems.

Recently, Ökcü and Aydıner studied the Joule-Thomson expansion process in the charged AdS black holes with the analogy between charged AdS black holes and van der Waals system[18]. For Joule-Thomson expansion in classical thermodynamics, gas at a high pressure passes through a porous plug to a section with a low pressure and during the process the enthalpy is unchanged. An interesting phenomenon during the Joule-Thomson expansion process is that the $T-P$ graph is divided into two parts, the cooling region and the heating region, by the inversion curve. The 
isenthalpy and inversion curves were first studied in charged AdS black holes and Kerr-AdS black holes[19]. Then the works were generalized to AdS black holes with a global monopole|20], higher dimensional AdS black holes[21], AdS black holes in Lovelock gravity [22] and charged Gauss-Bonnet black holes in AdS space[23], etc. All the articles above show that the inversion curves $T_{i}-P_{i}$ only have positive slope, which was different from the inversion curves in van der Waals system including both positive and negative slopes. However, black holes in previous discussion of JouleThomson expansion all exist singularity. In addition, Bardeen-AdS black holes have no singularity inside the horizon, called regular black holes. So, we would like to to see whether the singularity have the affect on the Joule-Thomson expansion for studying Bardeen-AdS black holes.

This paper is organized as follows. In Sec.II, we briefly review Bardeen-AdS black holes. In Sec.III, we investigate Joule-Thomson expansion for Bardeen-AdS black holes. The isenthalpic and inversion curves have been obtained. Conclusions and discussion are given in Sec.IV.

\section{A BRIEF REVIEW OF BARDEEN-ADS BLACK HOLES}

Bardeen black hole is known as a black hole without singularity [24] and the corresponding action with $\Lambda$ term can be given by

$$
S=\frac{1}{16 \pi} \int d^{4} x \sqrt{-g}\left[R+\frac{6}{l^{2}}-4 \mathcal{L}(F)\right],
$$

where $R$ is scalar curvature, $g$ is the determinant of the metric tensor, $l$ is AdS radius connected with $\Lambda$ through the relation $\Lambda=-\frac{3}{l^{2}} . \mathcal{L}(F)$ is a function of $F=\frac{1}{4} F_{\alpha \beta} F^{\alpha \beta}$ with $F_{\mu \nu}=2 \nabla_{[\mu} A_{\nu]}$ given by[25]

$$
\mathcal{L}(F)=\frac{3}{2 s q^{2}}\left(\frac{\sqrt{2 q^{2} F}}{1+\sqrt{2 q^{2} F}}\right)^{\frac{5}{2}} .
$$


The parameter $s$ in above equation is given by $\frac{|q|}{2 M}$ where $q$ and M correspond to the magnetic charge and the mass of the black hole. The field equations derived from the action in Eq. (11) are given by

$$
\begin{gathered}
G_{\alpha \beta}+\Lambda g_{\alpha \beta}=2\left(\frac{\partial \mathcal{L}(F)}{\partial F} F_{\alpha \lambda} F_{\beta}^{\lambda}-g_{\alpha \beta} \mathcal{L}(F)\right) \\
\nabla_{\alpha}\left(\frac{\partial \mathcal{L}(F)}{\partial F} F^{\beta \alpha}\right)=0 \\
\nabla_{\alpha}\left(* F^{\beta \alpha}\right)=0
\end{gathered}
$$

Then the line element of Bardeen-AdS black holes can be considered as

$$
d s^{2}=-f(r) d t^{2}+\frac{1}{f(r)} d r^{2}+r^{2}\left(d \theta^{2}+\sin ^{2} \theta d \phi^{2}\right)
$$

where $f(r)=1-\frac{2 m(r)}{r}$. Following the ansatz for Maxwell equation $F_{\mu \nu}=2 \delta_{[\mu}^{\theta} \delta_{\nu]}^{\phi} Z(r, \theta)[26]$ and with the help of Eq. (4), we can get

$$
F_{\mu \nu}=2 \delta_{[\mu}^{\theta} \delta_{\nu]}^{\phi} g(r) \sin \theta
$$

Using the condition $d F=g^{\prime}(r) \sin \theta d r \wedge d \theta \wedge d \phi=0$, we conclude that $g(r)=$ const. $=$ $g$. Hence, the field strength is $F_{\theta \phi}=-F_{\phi \theta}=q \sin \theta$ with $F=\frac{g^{2}}{2 r^{4}}$. Substituting the expression in Eq. (2), we get

$$
\mathcal{L}(F)=\frac{3 M g^{2}}{\left(g^{2}+r^{2}\right)^{\frac{5}{2}}} .
$$

Moreover, the field equation (3) can yield a solution for $m(r)$ with

$$
m(r)=\frac{M r^{3}}{\left(g^{2}+r^{2}\right)^{\frac{3}{2}}}-\frac{r^{3}}{2 l^{2}} .
$$

So the metric of Bardeen-AdS black hole is

$$
f(r)=1-\frac{2 M r^{2}}{\left(g^{2}+r^{2}\right)^{\frac{3}{2}}}+\frac{r^{2}}{l^{2}} .
$$


When $f\left(r_{+}\right)=0$, we get

$$
M=\left(1+\frac{r_{+}^{2}}{l^{2}}\right) \frac{\left(r_{+}^{2}+q^{2}\right)^{\frac{3}{2}}}{2 r_{+}^{2}},
$$

which satisfies the first law of black hole thermodynamics,

$$
d M=T d S+V d P+\Phi d q .
$$

Here the pressure $P$ in AdS black holes corresponds to the $\Lambda$ with

$$
P=-\frac{\Lambda}{8 \pi}=\frac{3}{8 \pi l^{2}}
$$

and the conjugate quantity $V$ of the pressure $P$ can be given by

$$
V=\left(\frac{\partial M}{\partial P}\right)_{S, q}=\frac{4 \pi r_{+}^{3}}{3}\left(1+\frac{q^{2}}{r_{+}^{2}}\right)^{\frac{3}{2}} .
$$

The Hawking temperature $T$ can be obtained from the first derivative of $f(r)$ at the horizon[27],

$$
T=\frac{\kappa}{2 \pi}=\frac{f^{\prime}\left(r_{+}\right)}{4 \pi}=\frac{3 r_{+}}{4 \pi\left(r_{+}^{2}+q^{2}\right)}+\frac{3 r_{+}^{3}}{4 \pi\left(r_{+}^{2}+q^{2}\right) l^{2}}-\frac{1}{2 \pi r_{+}} .
$$

Then, the equation of state $P=P(V, T)$ for Bardeen-AdS black holes can be obtained from Eqs. (13) and (15),

$$
P=\frac{4 \pi r_{+}\left(q^{2}+r_{+}^{2}\right) T+2 q^{2}-r_{+}^{2}}{8 \pi r_{+}^{4}} .
$$

According to [8], the critical temperature can be calculated by

$$
\frac{\partial P}{\partial v}=0, \quad \frac{\partial^{2} P}{\partial v^{2}}=0
$$

where $v$ is $\frac{2 r_{+}^{3}}{q^{2}+r_{+}^{2}}$ and we can get

$$
T_{c}=\frac{7+\sqrt{273}}{\sqrt{2(15+\sqrt{273})}(21+\sqrt{273}) \pi q} .
$$




\section{JOULE-THOMSON EXPANSION}

In this section, we will investigate Joule-Thomson expansion for Bardeen-AdS black holes. The main feature of this process is that enthalpy remains constant. In addition, the enthalpy $H$ can be identified as the mass $M$ of the AdS black holes [7]. So, we can keep the black hole mass constant. Similar to the Joule-Thomson process with fixed particle number for van der Waals gases, we consider the canonical ensenmble with fixed magnetic charge $q$. Therefore, Joule-Thomson coefficient $\mu$, which characterizes the expansion, is given by [23]

$$
\mu=\left(\frac{\partial T}{\partial P}\right)_{M, q}=\left(\frac{\partial T}{\partial r_{+}}\right)_{M, q}\left(\frac{\partial r_{+}}{\partial P}\right)_{M, q}
$$

From Eqs. (11) and (15), the pressure and temperature written as a function of $M$ and $r_{+}$are given

$$
\begin{aligned}
& P\left(M, r_{+}\right)=\frac{-3\left(q^{2}+r_{+}^{2}\right)^{\frac{3}{2}}+6 M r_{+}^{2}}{8 \pi r^{2}\left(q^{2}+r_{+}^{2}\right)^{\frac{3}{2}}} \\
& T\left(M, r_{+}\right)=\frac{-\left(q^{2}+r_{+}^{2}\right)^{\frac{5}{2}}+3 M r_{+}^{4}}{2 \pi r\left(q^{2}+r_{+}^{2}\right)^{\frac{5}{2}}}
\end{aligned}
$$

So, one can obtain the Joule-Thomson coefficient

$$
\begin{aligned}
\mu & =\frac{2 r_{+}\left(9 M q^{2} r_{+}^{4}-6 M r_{+}^{6}+\left(q^{2}+r_{+}^{2}\right)^{\frac{7}{2}}\right)}{3\left(q^{2}+r_{+}^{2}\right)^{\frac{7}{2}}\left(1-\frac{3 M r_{+}^{4}}{\left(q^{2}+r_{+}^{2} \frac{5}{2}\right.}\right)} \\
& =\frac{4 q^{4} r_{+}+q^{2}\left(26 r_{+}^{3}+48 \pi P r_{+}^{5}\right)-8\left(r_{+}^{5}+4 \pi P r_{+}^{7}\right)}{3\left(q^{2}+r_{+}^{2}\right)\left(2 q^{2}-r_{+}^{2}-8 \pi P r_{+}^{4}\right)} .
\end{aligned}
$$

Compare Eqs. (15) and (22), we can get that the divergent point of Joule-Thomson coefficient coincides with the zero point of temperature. The divergent point maybe reveals the information of Hawking temperature and corresponds to the extremal black hole.

The zero point of Joule-Thomson coefficient is the point that distinguishes the cooling 
process and the heating process. From Eq. (22), one can obtain that the inversion pressure $P_{i}$ and the corresponding $r_{+}$have the relation,

$$
2 q^{4}+q^{2}\left(13 r_{+}^{2}+24 \pi P_{i} r_{+}^{4}\right)-4\left(r_{+}^{4}+4 \pi P_{i} r_{+}^{6}\right)=0
$$

The only positive and real solution of the above function is so long that we will not list them here. But we depict the relation between the inversion temperature $T_{i}$ and the inversion pressure $P_{i}$ in Fig. 1 with the help of Eqs. (15) and (23). The inversion curves are not closed and there is only one inversion curve in comparison with van der Waals fluids. For low pressure, the inversion temperature decreases with magnetic charge $q$, whereas it increases with $q$ for high pressure.

Then we would like to probe the ratio between the minimum inversion temperature

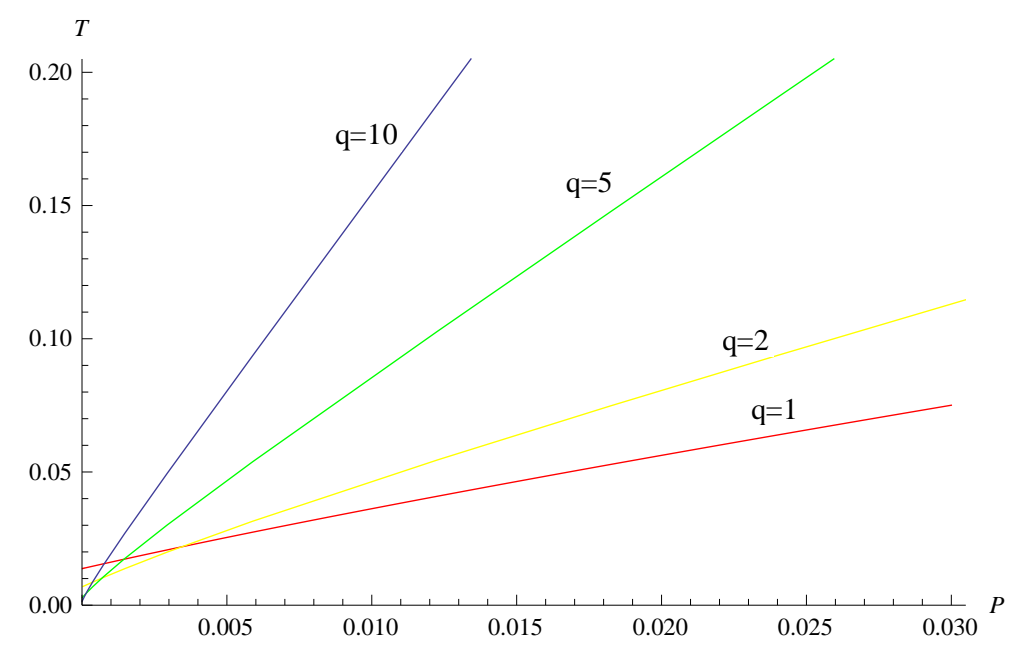

Figure 1. Inversion curves of Bardeen-AdS black holes in $T-P$ plane.

$T_{i}^{\text {min }}$ and the critical temperature $T_{c}$ first. Note that the minimum temperature can be obtained by demanding $P_{i}=0$. The Eq.(23) will be

$$
2 q^{4}+13 q^{2} r_{+}^{2}-4 r_{+}^{4}=0
$$


The only physically meaningful root is given by

$$
r_{+}=\frac{\sqrt{13+\sqrt{201}} q}{2 \sqrt{2}} .
$$

One can substitute the root into Eq. (15) and obtain the minimum inversion temperature,

$$
T_{i}^{\text {min }}=\frac{-3+\sqrt{201}}{\sqrt{2(13+\sqrt{201})}(21+\sqrt{201}) \pi q} .
$$

The ratio between minimum inversion and critical temperature is given by

$$
\frac{T_{i}^{\min }}{T_{c}} \approx 0.545874 .
$$

This ratio is not equal to the value in other black holes [18 22]. This different ratio may be due to the non-linear electrodynamics field and the correctional volume of Bardeen-AdS black holes. Moreover, we can substitute Eq. (25) into Eq. (11) and obtain the mass corresponding to the minimum inversion temperature,

$$
M_{\text {min }}=\frac{(21+\sqrt{201})^{3 / 2}}{4 \sqrt{2}(13+\sqrt{201})} q \approx 1.3571 q .
$$

This corresponding mass will help us to determine the black hole whether have the inversion point in Joule-Thomson expansion. The isenthalpic and inversion curves for various values of magnetic charge $q$ has been drawn in Fig. 2. As can been seen from Fig. 2, the inversion curves divide the plane into two regions. The branch below the inversion curves corresponds to the heating process with negative slope. And the branch above the inversion curves corresponds to the cooling process with positive slope. It is interesting to see in Fig. 2 that one isenthalpic curve does not have the inversion point. Moreover, for $q=10$ in Eq. (28), we have the mass $M_{\min } \approx 13.571$ which is greater than $M=13.5$. This also shows that the black hole with $q=10$ and $M=13.5$ does not have the inversion point. In other word, the black hole with $q=10$ and $M=13.5$ is always in heating process. 


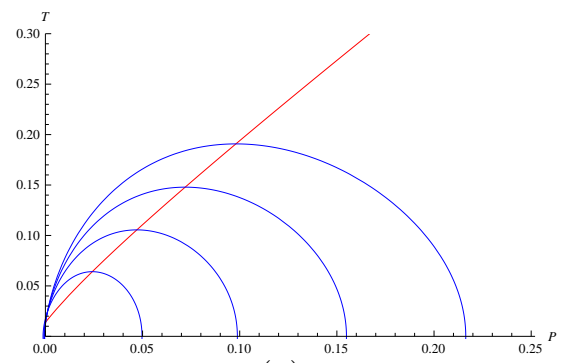

(a)

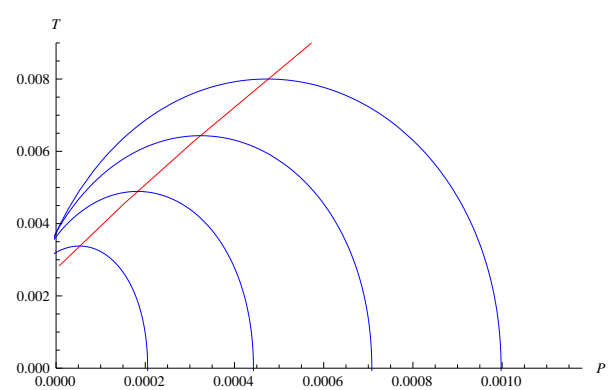

(c)

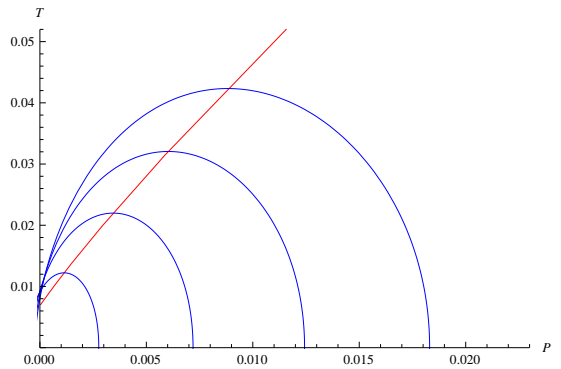

(b)

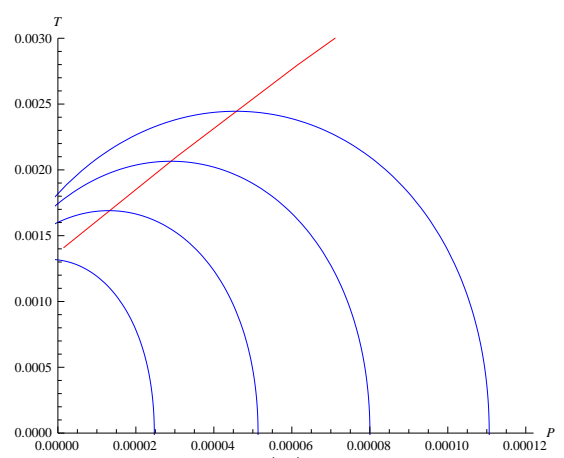

(d)

Figure 2. Inversion and isenthalpic curves of Bardeen-AdS black holes. The red lines present inversion curves and the blue ones are isenthalpic curves. From bottom to top, isenthalpic curves correspond to increasing values of $M$. (a) $q=1$ and $M=2,2.5,3,3.5$. (b) $q=2$ and $M=3,3.5,4,4.5$. (c) $q=5$ and $M=7,7.5,8,8.5$. (d) $q=10$ and $M=13.5,14,14.5,15$.

The same phenomenon can alao be obtained from the charged AdS black holes with large $Q$ which is not given in [18]. The figure we have plotted in Fig. 3. Similar steps can be used in charged AdS black holes[18], we get

$$
M_{\min }^{Q}=\frac{5 \sqrt{6}}{12} Q \approx 1.0206 Q
$$

Similarly, for $Q=35$ in Eq. (29), we have the mass $M_{\min }^{Q} \approx 35.722>35.5$. 


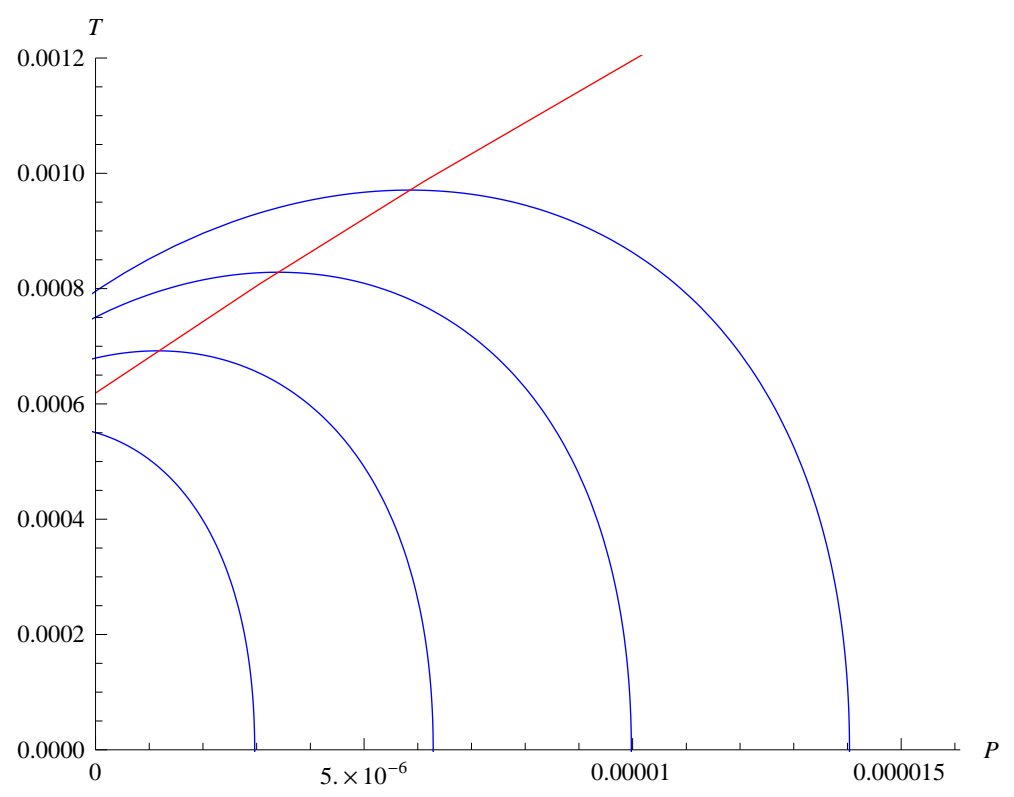

Figure 3. Inversion curves of charged AdS black holes in $T-P$ plane. $Q=35$ and $M=35.5,36,36.5,37$

\section{CONCLUSIONS AND DISCUSSION}

In this article, we studied Joule-Thomson expansion for Bardeen-AdS black holes in the extended phase space. We considered the expansion process with constant mass as the mass of AdS black holes was identified as enthalpy. Firstly, we got JouleThomson coefficient and found that the divergent point of Joule-Thomson coefficient coincided with the zero point of temperature. That is to say, the divergent point of Joule-Thomson coefficient reveals the information of Hawking temperature.

Then we plotted inversion curves with different magnetic charge $q$ and found there was only one inversion curve which was different from van der Waals fluids having two inversion curves. Moreover, we obtained the minimum inversion temperature $T_{i}$ and the corresponding mass. The ratio between minimum inversion and 
critical temperatures was calculated for Bardeen-AdS black holes. The ratio is not equal to the value which have been obtained in other articles and this different may be due to the non-linear electrodynamics field and the correction volume of BardeenAdS black holes.

We also plotted the isenthalpic and inversion curves in the $T-P$ plane. The inversion curve divides the $T-P$ plane into two regions. The region above the inversion curve is the cooling region with positive slope for isenthalpic curves. The region under the inversion curve is the heating region with negative slope for isenthalpic curves. An interesting phenomenon is that there is an isenthalpic curve does not have the critical point which means that the black hole is always in heating process. That is to say, as the pressure goes down, the temperature of horizon in this black hole can keep rising during the Joule-Thomson expansion. The mass of this black hole is also less than the mass corresponding the minimum inversion temperature. In addition, we also get the same phenomenon of charged AdS black holes with large charge $Q$. This phenomenon may be also obtained from other black holes.

\section{CONFLICTS OF INTEREST}

The authors declare that there are no conflicts of interest regarding the publication of this paper.

\section{ACKNOWLEDGMENTS}

We would like to thank the National Natural Science Foundation of China (Grant No.11571342) for supporting us on this work. 


\section{REFERENCES}

[1] Jacob D. Bekenstein. Black holes and entropy. Phys. Rev. D, 7:2333-2346, Apr 1973.

[2] J. M. Bardeen, B. Carter, and S. W. Hawking. The four laws of black hole mechanics. Communications in Mathematical Physics, 31(2):161-170, Jun 1973.

[3] S. W. Hawking. Particle creation by black holes. Communications in Mathematical Physics, 43(3):199-220, Aug 1975.

[4] S. W. Hawking and Don N. Page. Thermodynamics of black holes in anti-de sitter space. Communications in Mathematical Physics, 87(4):577-588, Dec 1983.

[5] Andrew Chamblin, Roberto Emparan, Clifford V. Johnson, and Robert C. Myers. Holography, thermodynamics, and fluctuations of charged ads black holes. Phys. Rev. D, 60:104026, Oct 1999 .

[6] Andrew Chamblin, Roberto Emparan, Clifford V. Johnson, and Robert C. Myers. Charged ads black holes and catastrophic holography. Phys. Rev. D, 60:064018, Aug 1999.

[7] David Kastor, Sourya Ray, and Jennie Traschen. Enthalpy and the mechanics of AdS black holes. Classical and Quantum Gravity, 26(19):195011, sep 2009.

[8] David Kubizňák and Robert B. Mann. P-v criticality of charged ads black holes. Journal of High Energy Physics, 2012(7):33, Jul 2012.

[9] Sharmila Gunasekaran, David Kubizňák, and Robert B Mann. Extended phase space thermodynamics for charged and rotating black holes and born-infeld vacuum polarization. Journal of High Energy Physics, 2012(11):110, 2012.

[10] Shao-Wen Wei and Yu-Xiao Liu. Critical phenomena and thermodynamic geometry of charged gauss-bonnet ads black holes. Physical Review D, 87(4):044014, 2013. 
[11] Shao-Wen Wei and Yu-Xiao Liu. Triple points and phase diagrams in the extended phase space of charged gauss-bonnet black holes in ads space. Physical Review D, 90(4):044057, 2014.

[12] Li-Chun Zhang, Meng-Sen Ma, Hui-Hua Zhao, and Ren Zhao. Thermodynamics of phase transition in higher-dimensional reissner-nordström-de sitter black hole. The European Physical Journal C, 74(9):3052, 2014.

[13] $\mathrm{SH}$ Hendi and MH Vahidinia. Extended phase space thermodynamics and $p-v$ criticality of black holes with a nonlinear source. Physical Review D, 88(8):084045, 2013.

[14] Jie-Xiong Mo and Wen-Biao Liu. Ehrenfest scheme for $p-v$ criticality of higher dimensional charged black holes, rotating black holes, and gauss-bonnet ads black holes. Physical Review D, 89(8):084057, 2014.

[15] David Kastor, Sourya Ray, and Jennie Traschen. Smarr formula and an extended first law for lovelock gravity. Classical and Quantum Gravity, 27(23):235014, nov 2010.

[16] Antonia M. Frassino, David Kubizňák, Robert B. Mann, and Fil Simovic. Multiple reentrant phase transitions and triple points in lovelock thermodynamics. Journal of High Energy Physics, 2014(9):80, Sep 2014.

[17] Natacha Altamirano, David Kubizňák, Robert B Mann, and Zeinab Sherkatghanad. Kerr-ads analogue of triple point and solid/liquid/gas phase transition. Classical and Quantum Gravity, 31(4):042001, 2014.

[18] Özgür Ökcü and Ekrem Aydıner. Joule-thomson expansion of the charged ads black holes. The European Physical Journal C, 77(1):24, Jan 2017.

[19] Özgür Ökcü and Ekrem Aydıner. Joule-thomson expansion of kerr-ads black holes. The European Physical Journal C, 78(2):123, Feb 2018.

[20] CL Ahmed Rizwan, A Naveena Kumara, Deepak Vaid, and KM Ajith. Joule-thomson expansion in ads black hole with a global monopole. International Journal of Modern 
Physics A, 33(35):1850210, 2018.

[21] Jie-Xiong Mo, Gu-Qiang Li, Shan-Quan Lan, and Xiao-Bao Xu. Joule-thomson expansion of $d$-dimensional charged ads black holes. Phys. Rev. D, 98:124032, Dec 2018.

[22] Jie-Xiong Mo and Gu-Qiang Li. Effects of lovelock gravity on the joule-thomson expansion. arXiv preprint arXiv:1805.04327, 2018.

[23] Shan-Quan Lan. Joule-thomson expansion of charged gauss-bonnet black holes in ads space. Phys. Rev. D, 98:084014, Oct 2018.

[24] James M Bardeen. Non-singular general-relativistic gravitational collapse. In Proc. Int. Conf. GR5, Tbilisi, volume 174, 1968.

[25] Sharmanthie Fernando. Bardeen de sitter black holes. International Journal of Modern Physics D, 26(07):1750071, 2017.

[26] Eloy Ayón-Beato and Alberto García. The bardeen model as a nonlinear magnetic monopole. Physics Letters B, 493(1):149 - 152, 2000.

[27] T Padmanabhan. Classical and quantum thermodynamics of horizons in spherically symmetric spacetimes. Classical and Quantum Gravity, 19(21):5387, 2002. 